\title{
Exploitation of InSAR techniques as a support of in situ sensors to improve safety and productivity in mining operations
}

\author{
J Duro DARES TECHNOLOGY, Spain \\ R Iglesias DARES TECHNOLOGY, Spain \\ D Monells DARES TECHNOLOGY, Spain \\ R Calvo DARES TECHNOLOGY, Spain
}

\begin{abstract}
The Brumadinho dam disaster occurred on 25 January 2019 when a tailings dam at the Córrego do Feijão iron ore mine in Brazil suffered a catastrophic failure of devastating consequences over hundreds of kilometres with a severe toll on the environment and the population. The collapse of Brumadinho tailings dam could be considered among the worst incidences related to mining operations in recent years. Unfortunately, it will not be the last tragedy of this kind. According to researchers at World Mine Tailings Failures, the risk of occurrence for these catastrophic events will increase in the near future.

Mining operations are typically monitored by employing sparse networks of in situ sensors (prisms, ground-based radars, piezometers, etc.). This information does not cover the totality of the possible areas affected by deformation. Furthermore, it is difficult to manage since it is obtained from different sources, whose integration and comparison become a complex task.

In this context, spaceborne synthetic aperture radar (SAR) interferometry (InSAR) technology represents an attractive alternative to complement the monitoring of mining operations due to its capabilities. On the one hand, spaceborne InSAR could provide measurements of terrain deformation with millimetric precision over wide areas. And on the other hand, the monitoring can be carried out regardless of day-night cycles or the weather conditions in the areas of interest.

In recent years, the InSAR technology has improved, allowing the exploitation of key indicators revealing precursors in the trend of deformation time-series. This paper takes Cadia and Brumadinho as test sites and shows the potential of cross-sections and inverse velocity analysis to complement in situ sensors for the rapid identification of critical deformations on tailing dams and slopes in open pits.

Finally, an example of an online platform is presented as a way of improving the user experience to exploit InSAR monitoring services jointly with in situ information over a map of reference. This provides the end-users with a single platform with all the monitoring data to make easier the spatio-temporal analysis of the deformation and the assessment of critical spots of deformation.
\end{abstract}

Keywords: mining, monitoring, stability, tailings, open pit, InSAR, radar

\section{$1 \quad$ Introduction}

The exploitation of satellite imagery represents an extremely useful tool to analyse data from local to regional scale at a lower cost compared with conventional in situ techniques. In this framework, radar measurements linked to spaceborne platforms are increasingly being used for the monitoring of a wide range of ground displacement phenomena.

This is the case of synthetic aperture radar (SAR), which allows obtaining all-weather, all-day reflectivity images with a fine spatial resolution. If SAR images are taken at different times, SAR interferometry (InSAR) techniques allow exploiting phase differences between multi-temporal pairs of SAR images in order to obtain 
displacement information of areas affected by deformation phenomena (Massonet \& Feigl 1998; Bürgmann et al. 2000) with millimetric precision (Gabriel et al. 1989).

SAR sensors allow measuring surface displacement phenomena due to different causes (slopes, tailings, dewatering, injection heave, etc.) and at different scales (faults, slope areas, entire mines, etc.). These measurements provide a high level of spatial detail and can be integrated with information coming from in situ sensors, to get a unified view of ground deformation effects and to better understand their causes. This is crucial to guarantee safety and efficiency in operations in different sectors such as mining, where geotechnical managers are increasingly using data collected by SAR sensors to detect precursors, prevent accidents and improve productivity in the following areas:

- Open pits: InSAR techniques can prevent slope failures in open pits, monitor local displacements to timely alert managers about potential problems, and to initiate slope management programs for failure mitigation.

- Tailings dams: Security of tailings facilities is now a recognised priority at a corporate level and the concept of sustainable mining is an accepted part of the modern industry, often required by regulators. The increasing focus on tailings dam safety brings with it an increasing awareness of the importance of a reliable monitoring program to confirm that the tailings dam is in a safe condition. InSAR measurements can provide alert warning systems to be timely informed about any potential dam failure.

- Waste dumps: Landslides, erosion and subsidence of waste dumps are a challenge for the mining industry because they could lead to environmental problems, which can have a negative impact on their surroundings.

- Leach pads: When heaping ore for leaching, the stacking heights can sometimes reach up to $100 \mathrm{~m}$, so an analysis of slope stability is of high importance. Leach pad failures can be caused by extreme heights, base pressures, or long-term chemical and biological degradation of ore.

\section{Methodology}

\subsection{From classical InSAR to multi-temporal InSAR}

InSAR consists of the combination of the phase information of two SAR images of the same illuminated scenario, acquired at different times. The result is known as interferogram, whose phase $\Delta \varphi_{\text {int }}$ is sensitive to the scene topography and deformation phenomena:

$$
\Delta \varphi_{\mathrm{int}}=\frac{4 \pi}{\lambda r_{0}} \frac{B_{n} \Delta r}{\tan \alpha}+\frac{4 \pi}{\lambda r_{0}} \frac{B_{n} \Delta h}{\sin \alpha}+\frac{4 \pi}{\lambda} \Delta \rho+\Delta \varphi_{A P S}+\Delta \varphi_{N}
$$

where:

$\Delta \rho \quad=\quad$ increments in deformation between two certain spatial points of the interferogram.

$\Delta r \quad=\quad$ increments in range distance between two certain spatial points of the interferogram.

$\Delta \mathrm{h} \quad=$ increments in height between two certain spatial points of the interferogram.

$\lambda=$ the nominal wavelength of the radar.

$B_{n} \quad=$ indicates the perpendicular baseline.

$r_{0}=$ the sensor-to-target distance, $\alpha$ the incidence angle.

$\Delta \varphi_{A P S}=$ accounts for the atmospheric artefacts (typically known as the atmospheric phase screen in the InSAR literature).

$\Delta \varphi_{N}=$ refers to the noise component of the interferometric phase. 
If we ignore for a moment the atmospheric and noise phase contribution to the interferometric phase, there are three main terms in Equation 1 that provide information about the SAR acquisitions. The first term is known as the flat-earth component. It is deterministic and depends on the acquisition geometry so it can be computed in advance and compensated from the interferometric phase. The second term is the topographic component that contains information about the elevation profiles of the area. Finally, the third term is the deformation component that provides information about subsidences and uplifts on the area.

If a ground deformation occurs during the acquisition period, the phase of the interferogram contains both the topographic and the deformation components. By using external information about the topography of the area such as an existing digital elevation model (DEM), the topographic phase component can be estimated and compensated for. The result of subtracting the topography from the interferogram is known as a differential interferogram, and the resulting phase can be directly related to ground deformation. One full cycle in the interferometric fringes represents a displacement equivalent to half the wavelength of the electromagnetic wave employed by the SAR sensor. Figure 1 illustrates the displacement retrieval process (assuming no significant atmospheric phase screen (APS) contribution) over a subterranean mine area in Central Catalonia, using two SAR images acquired with the C-band satellite Sentinel-1, with a temporal baseline of two months.

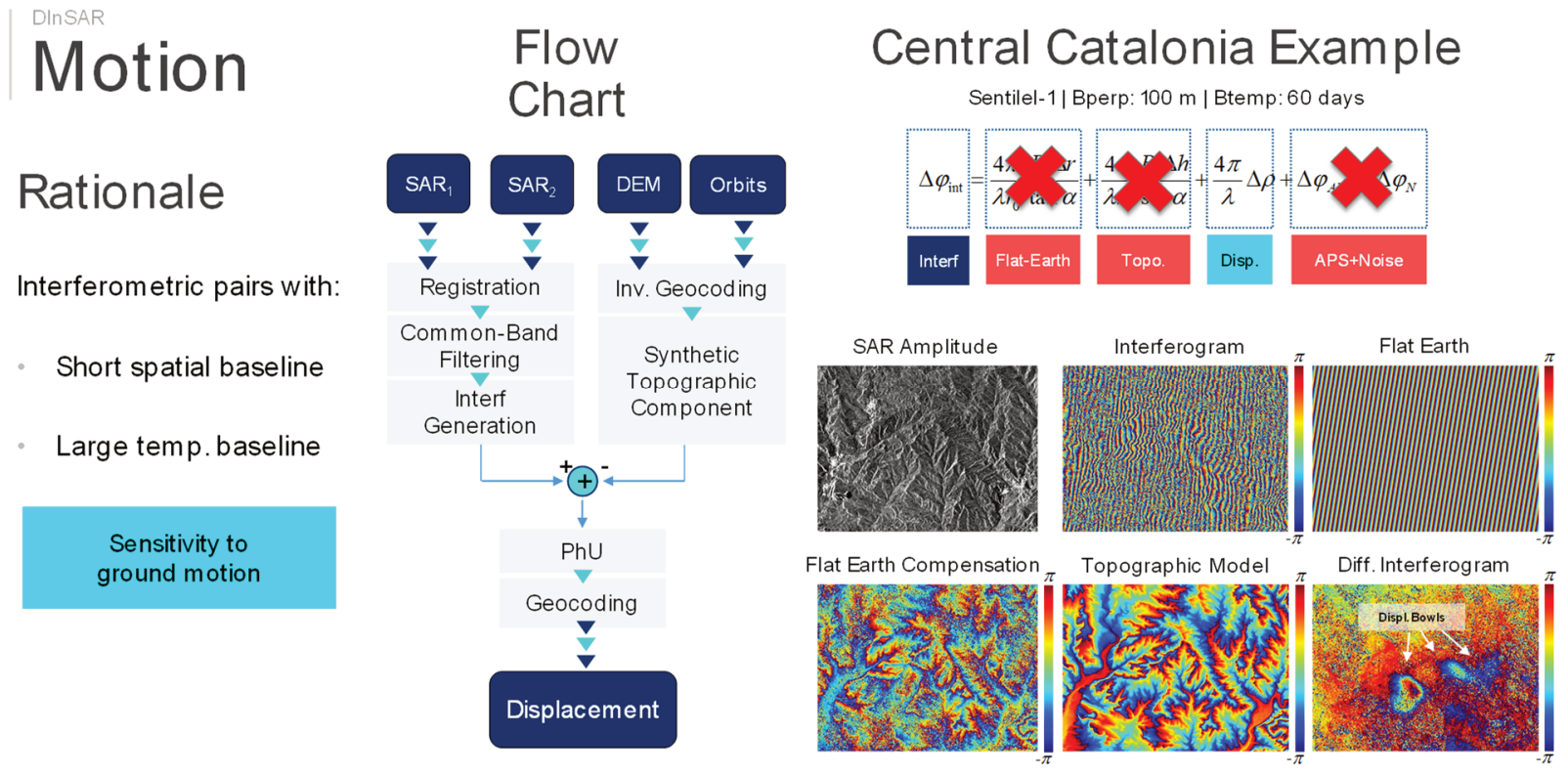

Figure 1 Displacement retrieval process over a subterranean mine area in Central Catalonia

Unfortunately, not all the points within the differential interferograms have enough phase quality to provide reliable information. Furthermore, the interferometric phase of differential interferograms is compromised by inaccuracies in the DEM employed during the compensation of the topographic component and, in general, by the impact of APS.

In order to compensate the topographic height errors and separate APS from displacement in differential interferograms, multi-temporal stacks of radar data are exploited using the so-called Persistent Scatterers Interferometry (PSI) technique. During the last 20 years, several PSI techniques have demonstrated their potential for measuring a wide range of deformation phenomena (Ferretti et al. 2001; Mora et al. 2002; Bernardino et al. 2002; Arnaud et al. 2003; Hooper et al. 2004; Lanari et al. 2004).

Figure 2 illustrates the objective of PSI, which is based on the identification of reliable measurement points in the illuminated scenario and in the compensation of phase-delay coming from atmospheric artefacts through the exploitation of multiple satellites passes. 

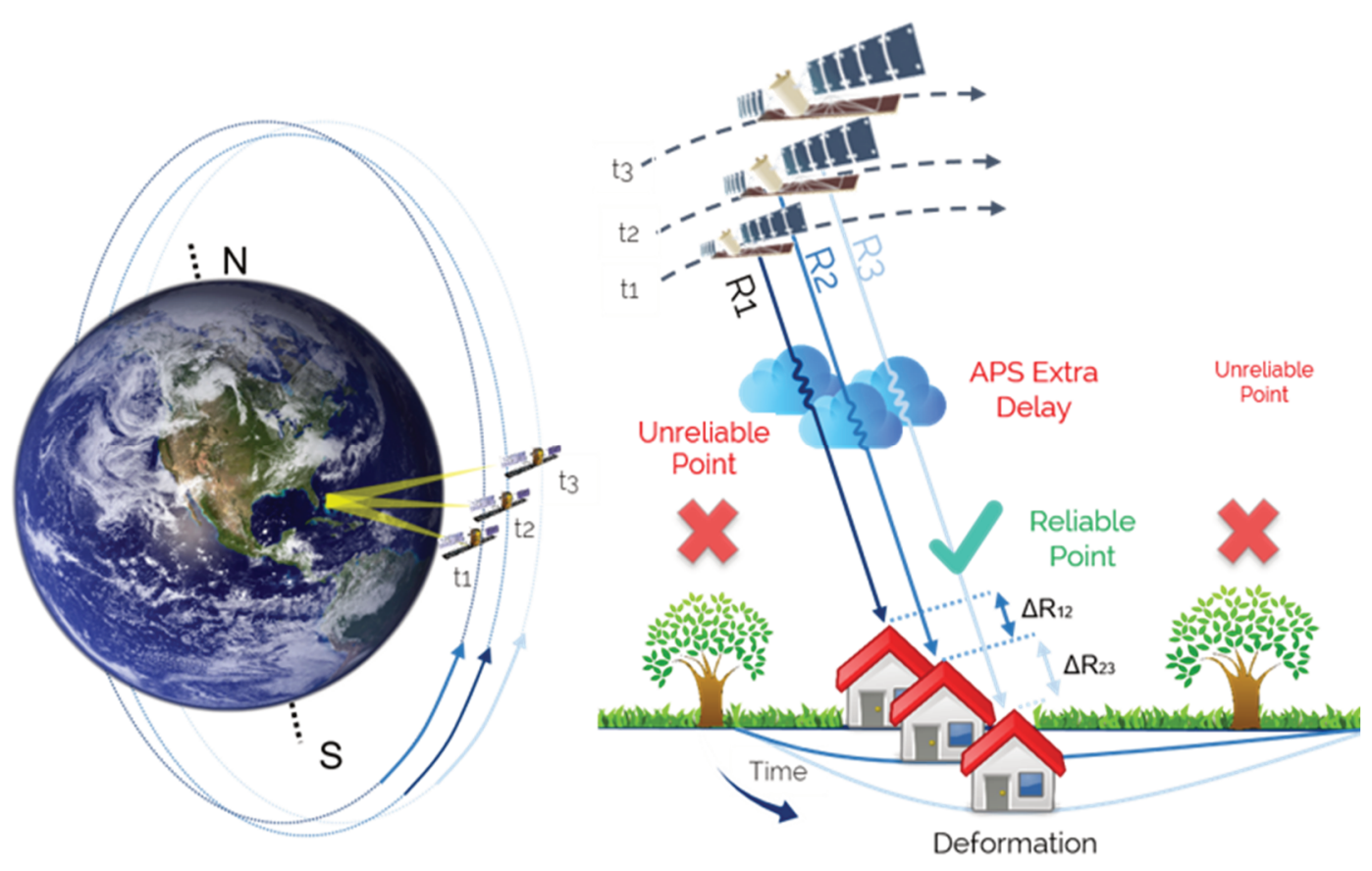

Figure 2 Persistent Scatterers Interferometry rationale

\subsection{The Coherent Pixels Technique}

Among the different PSI techniques available in the literature, DARES TECHNOLOGY employs the Coherent Pixels Technique (CPT) to obtain reliable ground displacement measurements. This PSI technique was developed in 2002 by the Remote Sensing Laboratory (RSLab) of the Universitat Politècnica de Catalunya (Mora et al. 2002). CPT allows the estimation of the linear and non-linear components of displacement, the topographic error, and the atmospheric artefacts.

In general terms, the CPT is divided into three main blocks, as described in Sections 2.2.1 to 2.2.3.

\subsubsection{Selection of differential interferograms and persistent scatterers}

The first block is focused on gettng a set of differential interferograms that are used to identify reliable scatterers (persistent scatterers). The persistent scatterers are points with high phase quality along the set of interferograms. These are the points that will be used in the PSI process to minimise the impact of decorrelation phenomena and ensure millimetric precision in the final ground displacement results.

\subsubsection{Linear estimation block}

Once a set of persistent scatterers is available, the estimation of the linear contribution of the differential phase is carried out. This is related to the linear component of ground displacement and the residual topographic error component remaining in the differential interferograms.

The first step of this block consists of doing a triangulation of the pixel candidates selected previously. This process works with phase increments between neighbouring pixels, instead of absolute phases. The objective of this approach is two-fold. On the one hand, atmospheric artefacts are minimised since they change smoothly in space in relation to the length of the links between neighbouring pixels. On the other hand, it cancels out the inherited phase offsets of each interferogram. The interferometric phase increment of each arc of the triangulation may be estimated with the following linear model:

$$
\Delta \varphi_{\text {mod }}=\frac{4 \pi}{\lambda} T \Delta v+\frac{4 \pi}{\lambda} \frac{B}{r_{0} \sin \alpha} \Delta \varepsilon+\Delta \varphi_{\text {res }}
$$


where:
$\Delta v \quad=$ the linear deformation rate in the line-of-sight (LOS).
$\Delta \varepsilon \quad=$ the vertical topographic error increment.
$\Lambda \quad=$ the wavelength.
$\mathrm{T}=$ the temporal spatial baseline.
B = the perpendicular spatial baselines.
$r_{0}=$ the sensor-to-target distance.
$\alpha=$ the incidence angle.
$\Delta \phi_{\text {res }}=$ accounts for the atmospheric, non-linear and noise components of the phase.

In order to estimate the linear deformation rate and the residual topographic error components of the interferometric phase, the model defined above is adjusted to the data through the minimisation of a cost function known as model adjustment function (MAF). With a suitable distribution of temporal and spatial baselines, the model can be correctly adjusted even in cases where some of the phase increments are wrapped since the solution is found in the complex plane. Once the linear deformation rate and the topographic error increments are obtained, the MAF is evaluated as a measurement of the quality of each arc. The low-quality arcs are truncated and at the same time, pixels that are isolated are removed. This process removes the pixels that do not fit the linear model, despite the fact that they have fulfilled the pixel selection thresholds. The objective of this step is cleaning those links largely affected by atmospheric artefacts. Finally, the absolute values of linear deformation and topographic error for each pixel are calculated through an integration process, using one or multiple seeds with known behaviour as tie points, leading to the a priori deformation results.

\subsubsection{Non-linear estimation block}

The non-linear estimation block compensates the atmospheric artefacts present in the interferograms and provides precise time-series of the displacements produced in the area of interest. First, the computed linear model is removed from the interferometric phase, so residual phases are generated. These remaining phases are supposed to be affected only by non-linear displacement and atmospheric artefacts. The separation of these components is based on a filtering process, taking advantage of their different temporal and spatial behaviour in a large set of images. Atmospheric artefacts are characterised by having a low spatial frequency behaviour for each interferogram, due to its roughly $1 \mathrm{~km}$ correlation window (Hanssen 2001). Regarding its temporal behaviour, for a given pixel (belonging to an image, not to an interferogram), it can be considered as a white process, since atmospheric conditions change randomly for typical acquisition intervals of current spaceborn SAR systems (6-20 days). Contrarily, non-linear displacement terms are considered to present a narrower spatial correlation window compared with APS and to behave low-pass along the temporal axis. The separation of the atmospheric artefacts and the non-linear component of displacement can hence be achieved, carrying out a filtering process in both the spatial and the temporal domains. In order to apply the temporal filtering, phase residues must be unwrapped, i.e. convert phase cycles into absolute phase values. Since a large number of fringes has been removed during the linear block, the phase unwrapping is supposed to be easier at this stage. Finally, since the process is based on the spatio-temporal behaviour of the deformation and the APS, the larger the number of images, the better the estimation of both components.

\subsection{Heading}

Figure 3 shows an example of ground displacement results and time-series over a mine in the Atacama Desert, Chile, using six months of data gathered by the European Space Agency Sentinel-1 (S1) satellite in descending mode. Red points refer to displacements moving away from the radar while blue ones indicate points approaching it. 
As shown in the figure, thanks to the global view of radar satellites, whole mines can be monitored with millimetric precision. In this case, slope stability over pit areas, settlement of waste dumps and subsidence in infrastructures areas and tailings dam can be clearly appreciated.

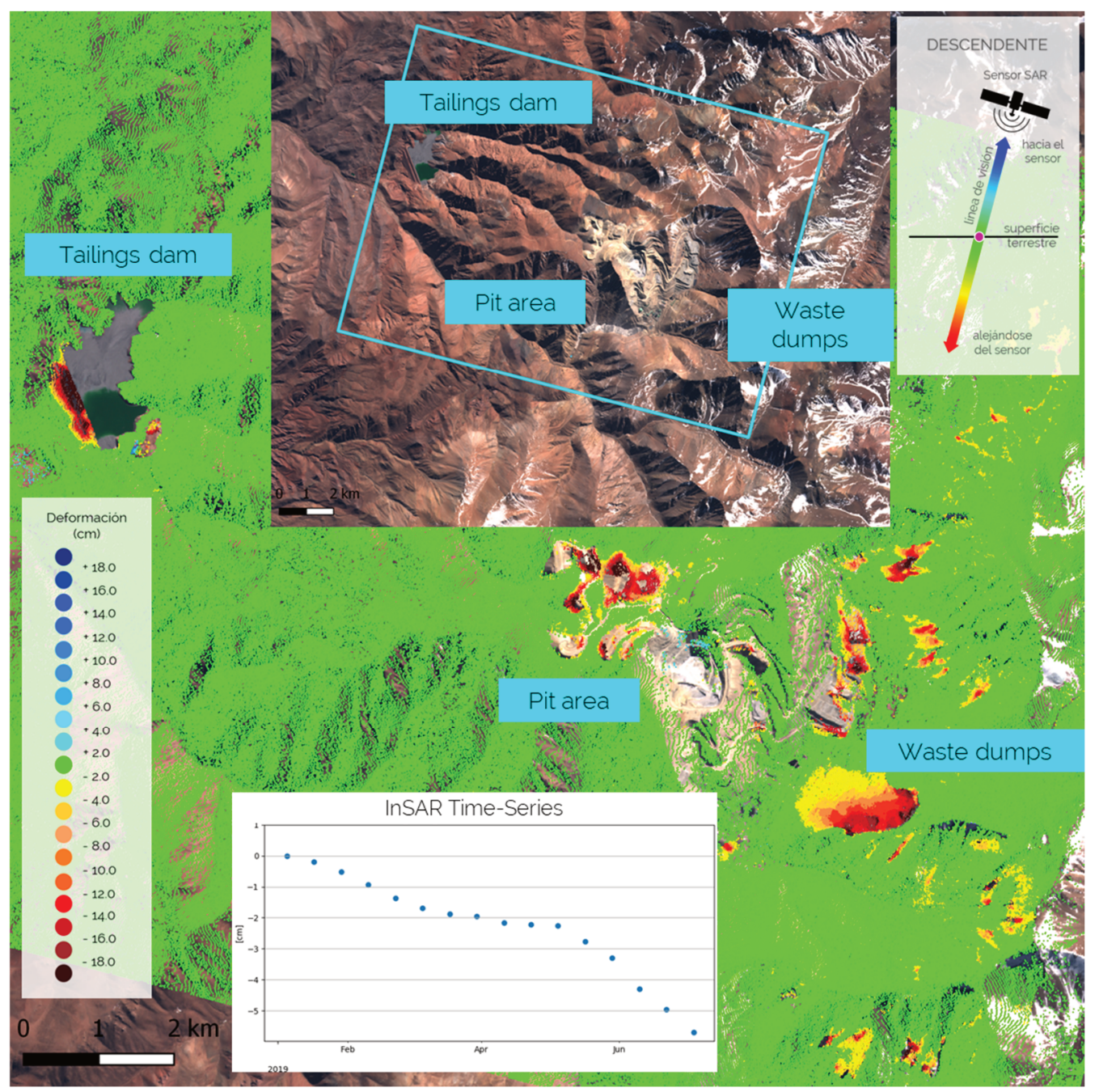

Figure 3 Persistent Scatterers Inferometry results at a mine in the Andes, South America, using data collected by the European Space Agency Sentinel-1 (S1) satellite. Red points refer to displacements moving away from the radar while blue ones indicate points approaching it. InSAR time-series show the evolution of ground deformation trough the time, allowing identification of acceleration periods

Despite the wide coverage and the large number of measurements shown, PSI products can be highly improved. On the one hand, mid-term reports are not enough to prevent failure events such as the one that occurred in Cadia gold mine on 9 March 2018 or in Brumadinho on 25 January 2019, since a frequent update of information is required. On the other hand, PSI standard products can be difficult to manage and to understand, especially over locations with topographic changes such as mining areas.

\subsection{From conventional Persistent Scatterers Interferometry results to advanced products for mine industry}

First of all, the retrieval of the complete magnitude of displacement is proposed. SAR sensors are only sensitive to displacements produced along the LOS direction and therefore, the measured displacements are a projection of the total magnitude. For the current polar-orbiting SAR sensors, the look direction is either east or west, for the ascending or descending pass respectively. Hence, SAR sensors are only sensitive to vertical displacements and/or horizontal ones along the east or west direction. If the displacement obtained 
by both acquisition modes ( $V_{L O S, A S C}$ and $\left.V_{L O S, D S C}\right)$ are combined, the up-down (UD) and east-west (EW) displacement components, $V_{U D}$ and $V_{E W}$, can be computed. With the incidence angle $\alpha$ and the track heading $\delta$ of both geometries, UD and EW components can be obtained solving the following system of linear equations (Hanssen 2001):

$$
\left(\begin{array}{ll}
-\cos \left(\alpha_{\mathrm{ASC}}\right) & \sin \left(\alpha_{\mathrm{ASC}}\right) \times \cos \left(\delta_{\mathrm{ASC}}\right) \\
-\cos \left(\alpha_{\mathrm{DSC}}\right) & \sin \left(\alpha_{\mathrm{DSC}}\right) \times \cos \left(\delta_{\mathrm{DSC}}\right)
\end{array}\right)\left(\begin{array}{c}
V_{U D} \\
V_{E W}
\end{array}\right)=\left(\begin{array}{c}
V_{L O S, A S C} \\
V_{L O S, \mathrm{DSC}}
\end{array}\right)
$$

Once the UD and EW components of displacement are obtained, a good magnitude of the displacement is generally computed from the modulus of both components.

The following advanced products can be now defined:

- Rapid reports: the generation of rapid reports through the computation of classical InSAR is firstly proposed. Instead of using PSI-derived displacement time-series, a single differential interferogram is generated with each satellite pass in order to identify any increase in deformation magnitude and/or in the shape of deformation areas with respect to the previous image. The precision of this product is, i.e. around one twentieth of the wavelength (which corresponds to $0.5 \mathrm{~cm}$ for sensors at C-band such as S1). Although it is not the highest precision that InSAR can achieve, it is extremely useful to identify new areas of deformation.

- Acceleration indicators and cross-sections: once hot spots of deformation are identified with rapid reports, PSI time-series can be analysed in detail. The problem at this point is that checking the large number of points provided by InSAR techniques can be a difficult task, sometimes impossible to fulfil. The computation of the acceleration over the last images of the time-series is proposed in this paper in order to highlight the potentially vulnerable areas in terms of increasing ground instabilities. Furthermore, the computation of cross-sections in these areas allows for spatio-temporal analysis of ground deformation along specific spatial profiles. An increase in the separation between consecutive graphs indicates an acceleration in the deformation process.

- Moreover, further analysis can be done in order to predict potential failures. The so-called inverse velocity approach (Carla et al. 2018) allows forecasting of the date of failure under a purely kinematic point of view (without accounting for the geology or the geotechnical features of the site). First, the velocity is computed as a derivative of the displacement $v=\frac{d \rho}{d t}$, and then a linear regression over the inverse velocity $\frac{1}{v}$ is calculated in order to find the intersection point with zero in the temporal axis. The potential failure date can be hence obtained through the coefficients of the linear regression.

- Visualisation and integration services: finally, the reporting of InSAR-derived products with in situ information is proposed. In this framework, webGIS services represent an excellent alternative to allow end-users to easily zoom over detailed optical images as background, and precisely identify ground deformation areas and compare them with in situ information. Finally, the computation of prediction indicators or the generation of transects can be easily incorporated into these services.

\section{$3 \quad$ Results and discussion}

\subsection{Rapid reports and cross-sections}

Mid-term reports represent a useful tool to identify areas with significant accumulated deformation. This is generally used by the survey team to identify potential field areas for detailed in situ inspections.

In order to overcome this limitation, rapid reports allow the detection of areas susceptible to structural problems by analysing the last satellite acquisitions. This advanced product allows a weekly or bi-weekly 
update to detect relevant time-series of risky areas and to timely inform managers about any potential change in the deformation magnitude and/or extension.

To provide the identification of hot spots with deformation, DARES TECHNOLOGY deliveries are based in a set of isolines in the areas affected by displacement. This way of presenting results eases the assessment of the areas affected by ground motion. No data areas (mainly due to geometric distortions) are indicated with a grey mask. The rest of the areas (without isolines) present stability.

Figure 4 corresponds to a rapid report example of the open pit area of a mine in Chile in late 2019 using the S1 satellite with a revisiting time of 12 days. The figure shows the total accumulated ground deformations over the last 12 days by the rapid reports, checking ground deformation increase over any area. The left-top and middle-top images present the motion in the ascending and descending LOS components, respectively. Through the decomposition approach described in Section 2.4, the corresponding UD and EW components can be obtained, represented in the left-bottom and middle-bottom images. The analysis of the decomposition reveals a dominant west motion detected in the northeast wall of the pit area. The main image on the right shows the total deformation, which is the magnitude of the EW and UD motion vector, directly related to the total magnitude of the motion present in the area. Displacement time-series taking into account one year of data in the area is presented showing an accumulated displacement of more than $2 \mathrm{~cm}$ during the last 12 days.
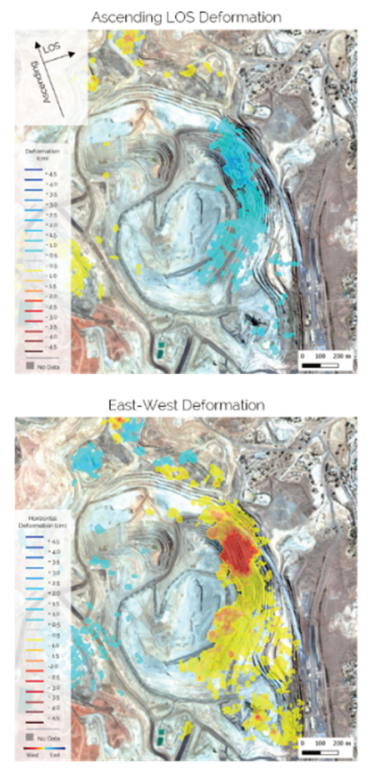
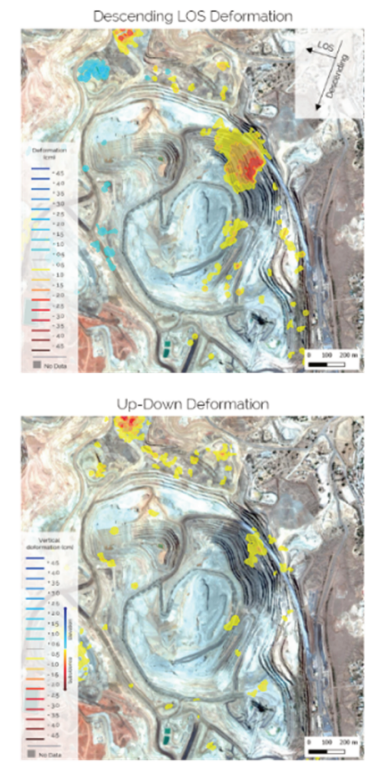

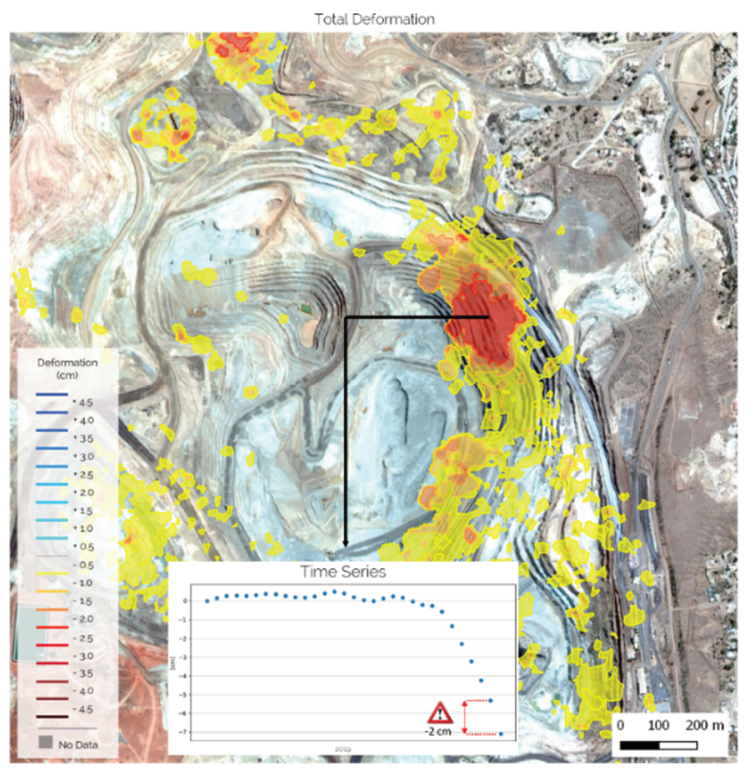

Figure 4 Rapid report example of the open pit area of a mine in Chile in late 2019, using S1 satellite. The left-top and middle-top images present the motion in the ascending and descending line-of-sight components, respectively. The left-bottom and middle-bottom images present the up-down (UD) and east-west (EW) displacement components, respectively. The main image on the right shows the total deformation, which is the magnitude of the EW-UD motion vector

The comparison of rapid reports in time can help in detecting the geotechnical precursors in mining areas. It can, therefore, help decision-makers to make better decisions about mine operations. However, further information can be retrieved from data to help in understanding the deformation dynamics exploiting multi-temporal data.

In order to include the temporal axis in the analysis of the areas of interest, the generation of cross-sections is proposed in this paper. An increase in the separation between consecutive transects is related to an acceleration in the deformation process.

Figure 5 shows a cross-section over a tailings dam located in the USA. The deformation dynamics cover a time period of one year (from April 2018 to April 2019) and including a period of heavy rains. The figure shows a clear acceleration followed by a deceleration with the arrival of autumn. 

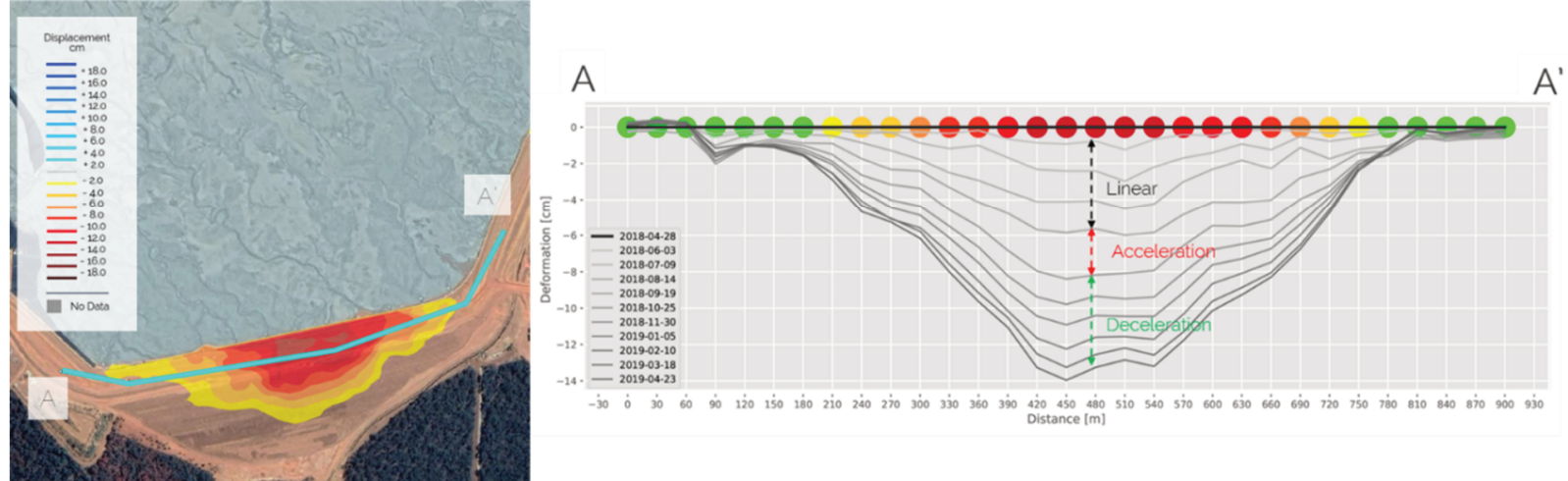

Figure 5 Cross-section over a tailings dam located in the USA. The increase in the separation between consecutive graphs (which are equally spaced in time) of the cross-section indicates an acceleration during August 2018 (shown as acceleration in the graph), coinciding with a period of heavy rains

The right part of Figure 5 shows deformation values for each satellite acquisition date. It shows accumulated deformations, showing the first reported deformation at the top and the last deformation line at the bottom of the cross-section. Please note cross-section from A to A' (as can be seen in the left side of the figure). Red dots correspond to the most severe deformation of the cross-section and green dots are stable points with no deformation in the period.

Another interesting advantage of using cross-sections is the ability to spot different behaviours in a complex scenario. An example is shown in Figure 6 corresponding to a cross-section of an open pit in a Chile mining area. It is a bi-annual report and it shows accumulated deformations that occurred in a two-year period. Profile $A A^{\prime}$ begins at the top of the northwest wall and ends at the bottom of the pit, crossing different areas of deformation. The corresponding cross-section reveals different acceleration behaviours depending on the area. Profile BB' $^{\prime}$ is drawn following one of the terraces of the open pit, with two main areas of deformation separated by a stable area due to the presence of a fault.

\section{Accumulated Biannual Displacement}

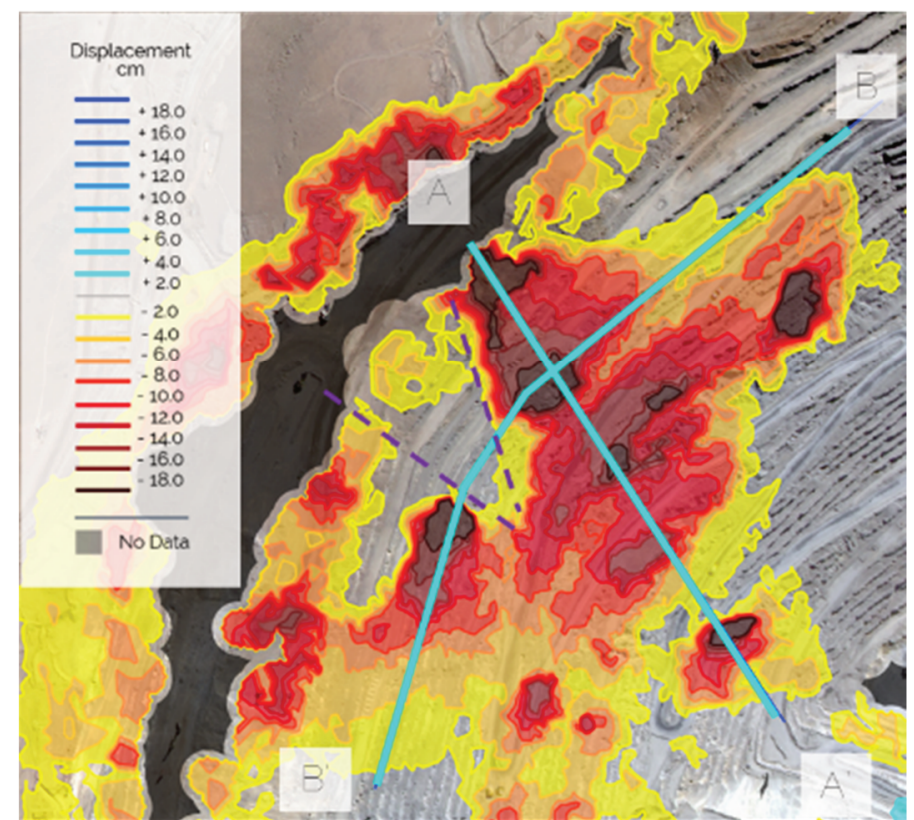




\section{Cross-Sections}

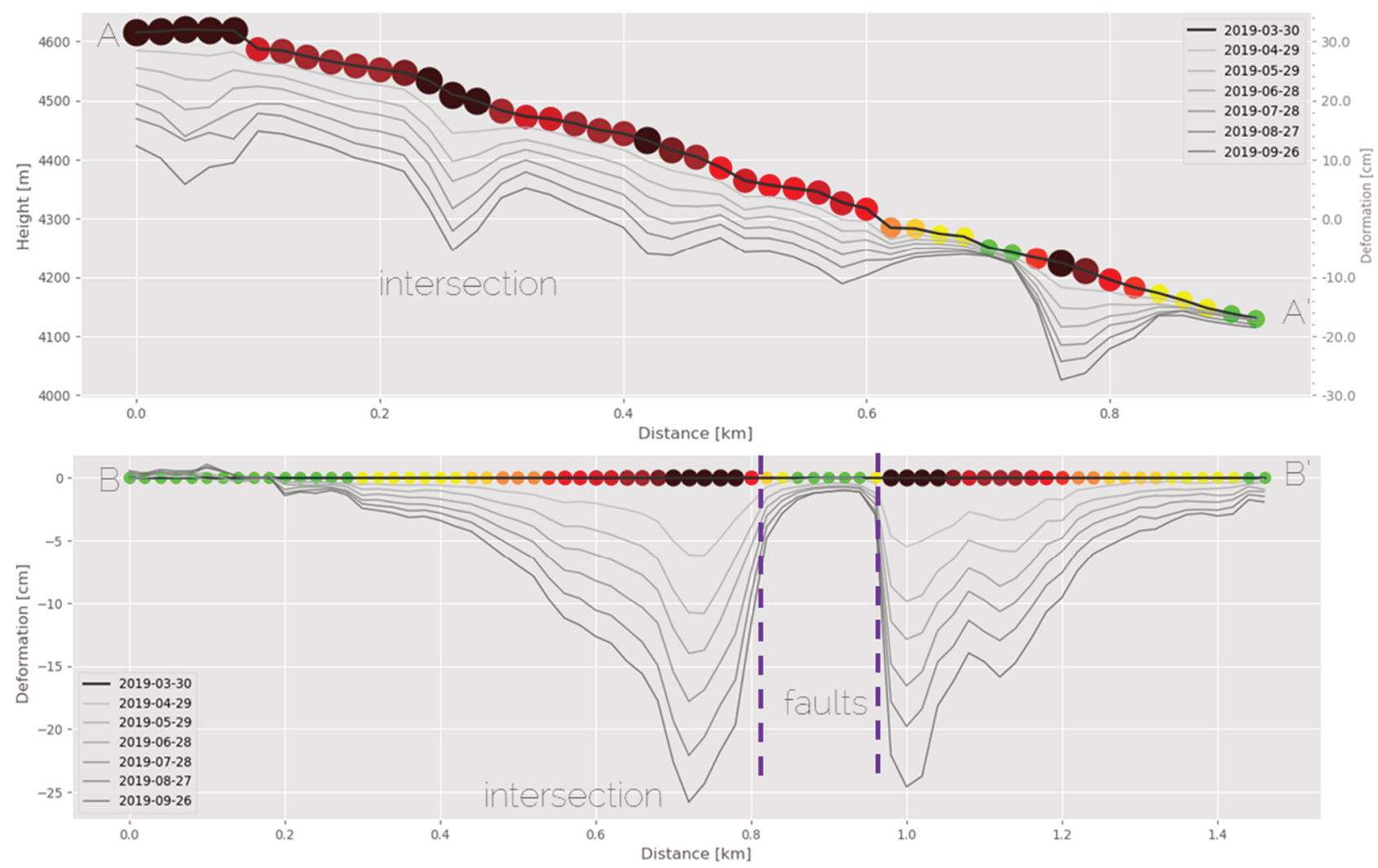

Figure 6 Cross-sections over an open pit located in an open pit mine in South America. The identification of an area of deformation separated by a stable area is related to the presence of a fault in the area

\subsection{Prediction indicators}

At this stage, the analysis of mine areas allows for the detection of deformation increase between consecutive acquisitions and its spatio-temporal behaviour is presented. The problem at this point is that checking the large number of points provided by InSAR products is a difficult task. For this reason, the next step is to look for time-series presenting an acceleration in the last period in order to detect behaviours that may lead to potential failures. In this framework, the computation of an acceleration indicator is proposed.

In order to illustrate the utility of this indicator, the back-analysis of Cadia gold mine in Australia, which suffered a tailings dam collapse on 9 March 2018, is presented. In Figure 7, the accumulated displacement measured before the event (from December 2017 to February of 2018) is superimposed over an optical image. Through the computation of an acceleration index over the time-series, the area that finally collapsed is clearly highlighted, showing a high concentration of points experiencing an acceleration in the last period. Acceleration means that the deformation evolution over time (time-series) shows a non-linear evolution, increasing the velocity of deformation over time. 
Rapid-Report 27/12/2017-25/02/2018

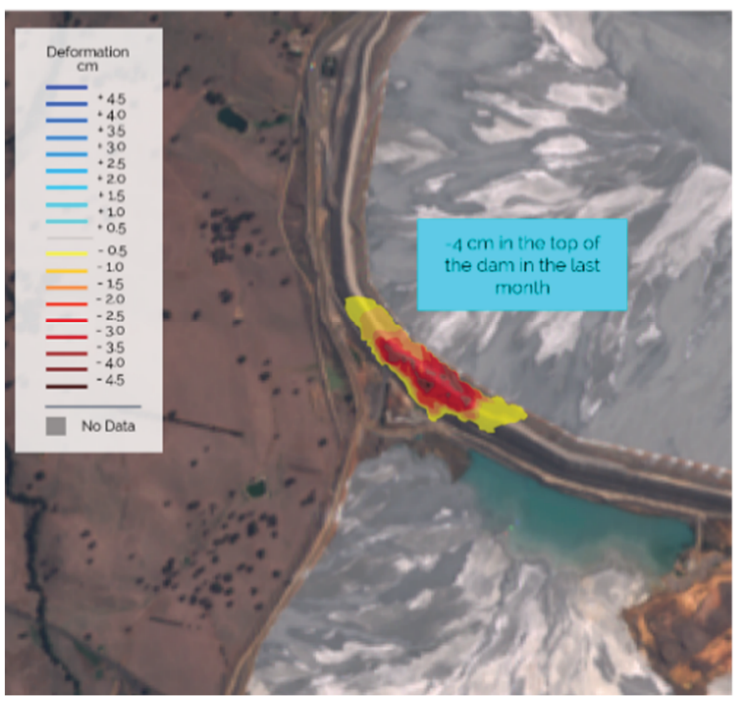

Acceleration Index

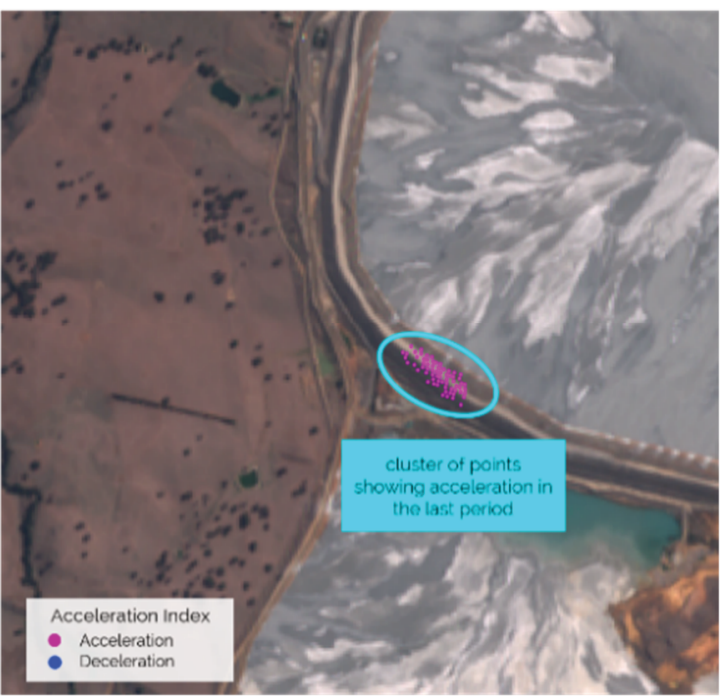

Figure 7 Rapid report and an acceleration index over Cadia mine tailings dam made in back-analysis thanks to archive satellite data available

As explained in the previous section, an inverse velocity analysis can be carried out in order to forecast the potential date of failure. This analysis can be performed over the areas highlighted by the acceleration index, thus providing an extremely useful early warning system to help managers in the decision-making process. In the back-analysis of Cadia presented, a prediction with four days of error is reached (see Figure 8).

Acceleration Index

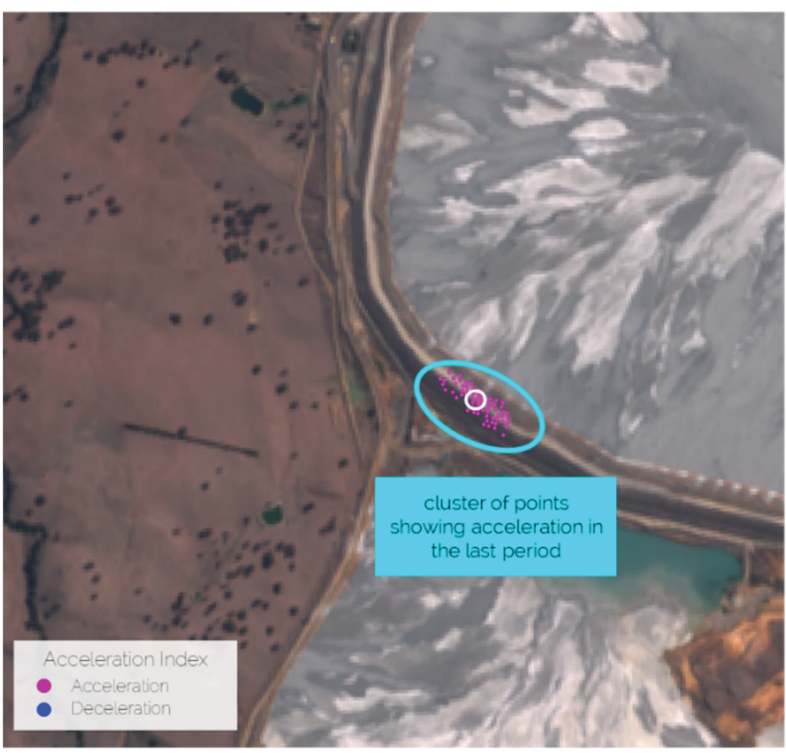

Inverse Velocity Analysis

Time-Series
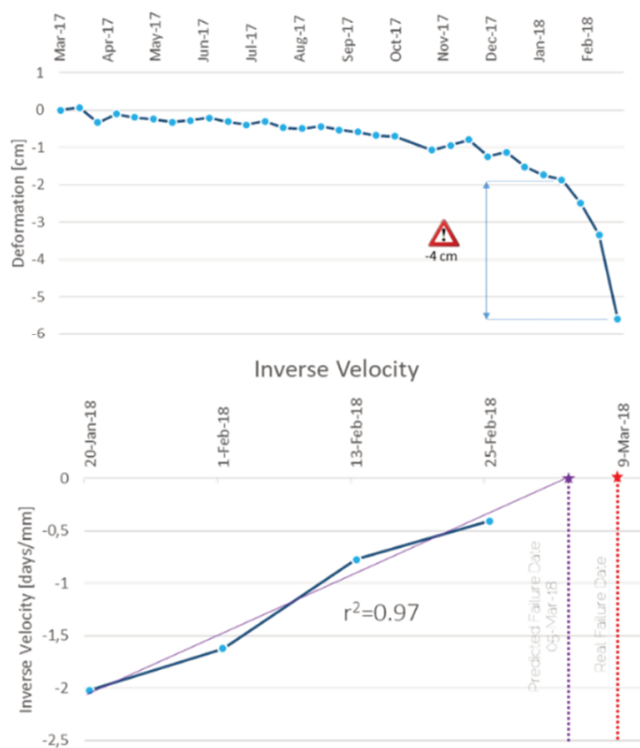

Figure 8 Acceleration index and inverse velocity back-analysis of the Cadia mine tailings dam failure using the measurement point highlighted with a white circle

Another interesting example is the back-analysis of the Brumadinho dam disaster that occurred on 25 January 2019, when a tailings dam at the Córrego do Feijão iron mine, 9 km (5.6 mi) east of Brumadinho, Brazil, suffered a catastrophic failure. The dam released a mudflow that advanced through the mine's offices, including a cafeteria during lunchtime, houses, farms, inns, and roads downstream. At least 237 people died as a result of the collapse. As seen in Figure 9, results show a displacement rate reaching up to $3 \mathrm{~cm} /$ year in the top of the dam. 
It is more interesting that another area of the dam is reaching higher velocities (of up to $-4 \mathrm{~cm} /$ year) in an area located at its base. Time-series analysis shows how this area experiences a clear acceleration at the end of 2018. Notice that the period of acceleration started about one and a half months before the collapse. An inverse velocity analysis has been used to determine whether the time of failure could have been predicted (28 January 2019). In this case, a prediction with three days of error is reached.

Displacement Rate

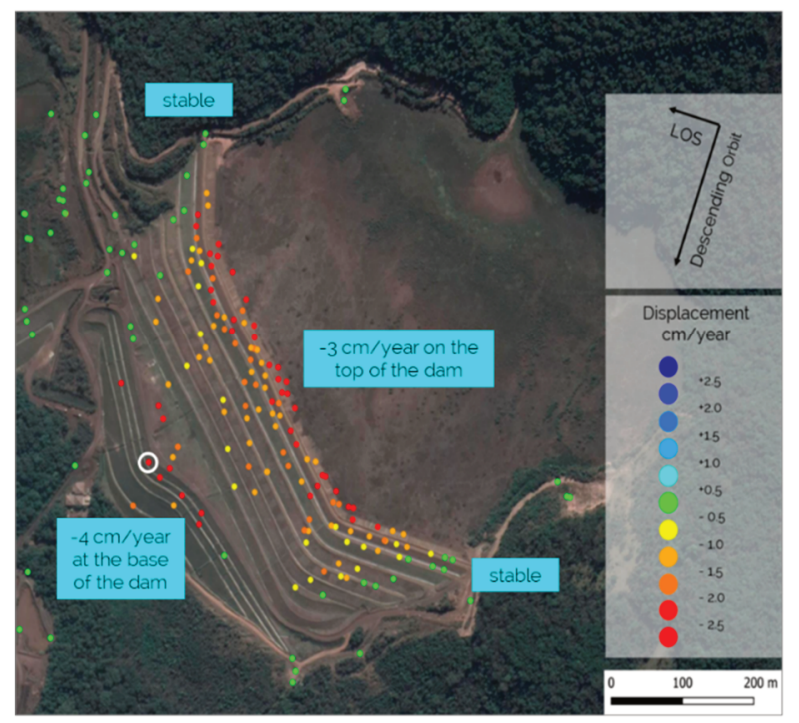

Inverse Velocity Analysis

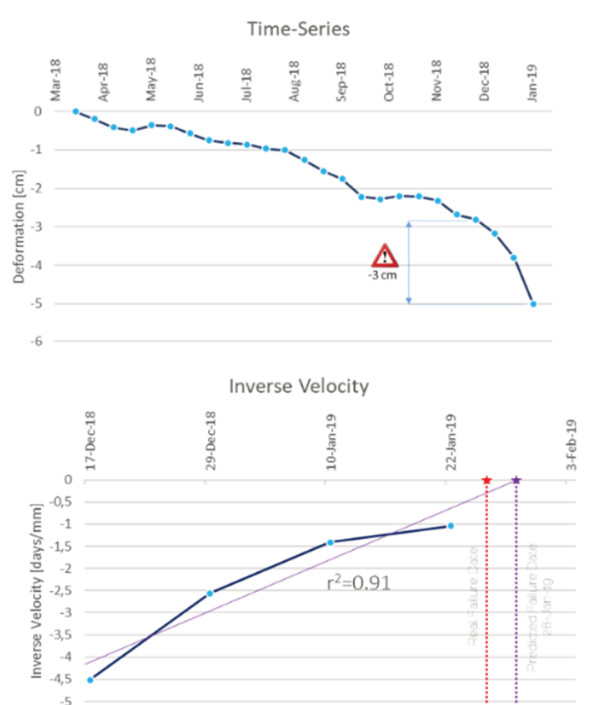

Figure 9 Displacement rate and inverse velocity back-analysis over the Brumadinho mine tailings dam failure using the measurement point highlighted with a white circle

Finally, the same analysis has been applied over an open pit failure in a gold mine in the USA (see Figure 10). The objective of this analysis is to demonstrate the capabilities of InSAR techniques to detect precursors in open pit areas. For confidentiality reasons, neither the location of the mine nor the failure date can be revealed, with errors of five days in the estimated potential failure date.

Acceleration Index

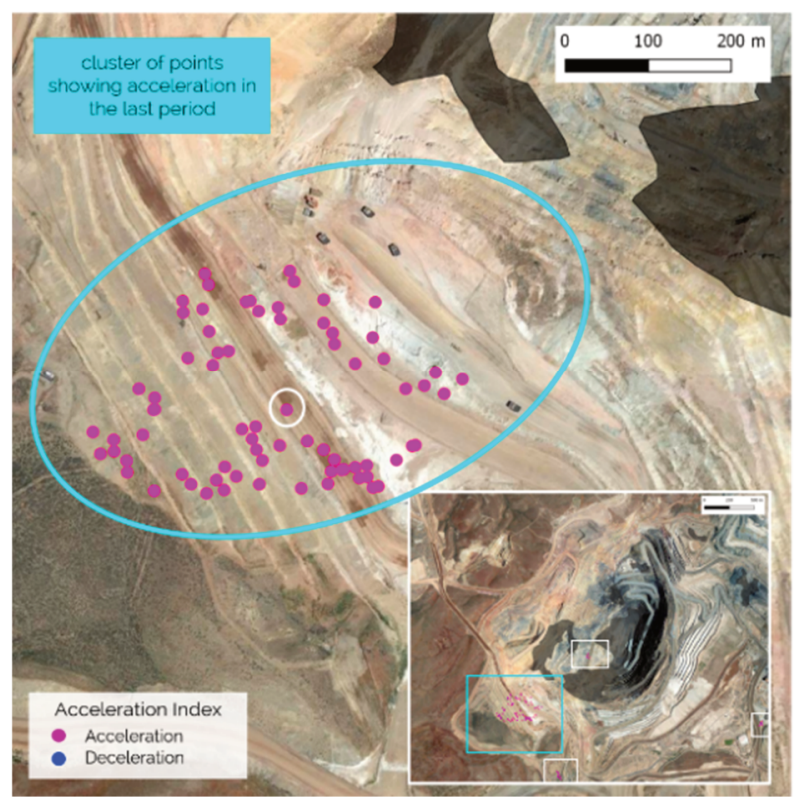

Inverse Velocity Analysis
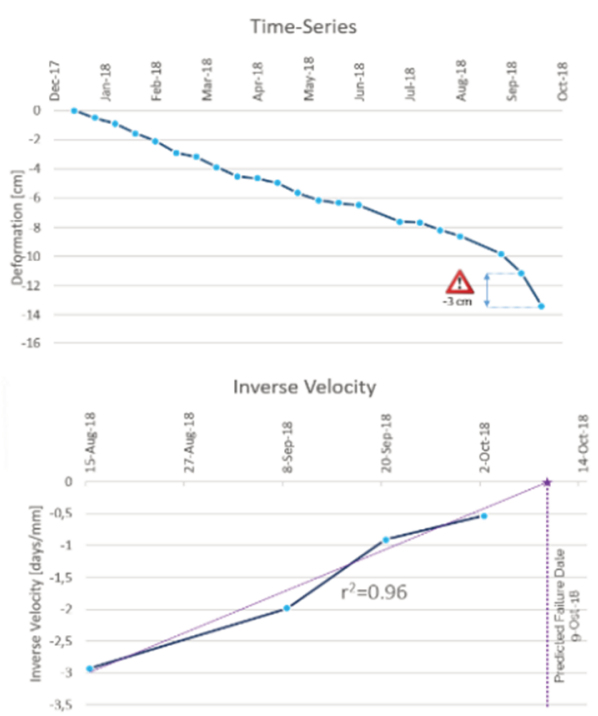

Figure 10 Acceleration index and inverse velocity back-analysis over an open pit mine in the USA. The graphs on the right side of the image correspond to the points inside the white circle on the left side of the image 


\subsection{Web-based visualisation tools and correlation with in situ sensors}

Nowadays, there is a lot of geotechnical data gathered through in situ instrumentation, models or remotely (ground radar, laser scanner, satellite InSAR, etc.). Indeed, in situ instrumentation provides valuable information to be exploited in an integrated scenario with other data providing multiple benefits towards a transversal approach. The management and automatic update of large databases of geotechnical data for the cross-correlation and the integrated analysis of multiple sources is one of the major challenges.

The combination of all InSAR products shown in the previous sections with in situ information clearly helps in the decision-making process by helping to identify potential risk areas and the cause-effect of unstable slopes in open pits and tailings dam.

For instance, piezometric measurements prove to be an important asset in the previously described scenario where the dynamics of water or fluids induce surface motion in the operations area. The simultaneous and near-real-time access to surface dynamics and piezometric level variations can detect the correlation between both the surface motion and the cover dynamics with the water level variations. Derived from this, correlation susceptibility maps or heatmaps can be derived to emphasise the relevant areas that can be affected by future events. Data from other sources of information, such as ground-based radar, GPS, total stations, meteorological data, PRISMs, piezometers, extensometers, etc., can be integrated with InSAR products.

Finally, the reporting of InSAR services with in situ information can benefit from webGIS services. In this framework, a solution for integrating all the geotechnical information is presented (see Figure 11). Online interactive webGIS InSAR services with in situ data integration improves conventional reporting (reports, emails, etc.), allowing end-users to easily zoom in and out over a detailed optical image as background in order to precisely identify where ground deformation is happening and to make further analysis comparing this information with in situ sensors, computing prediction indicators or generating transects over affected areas.
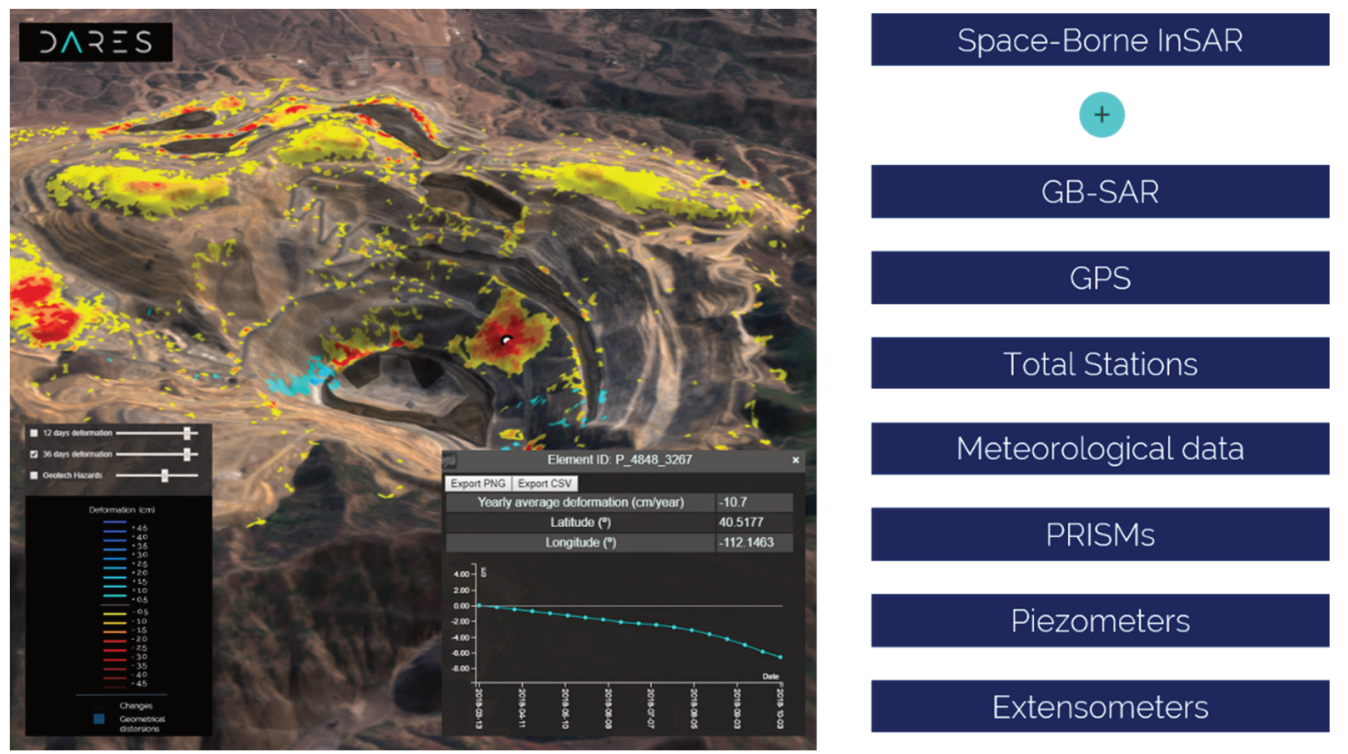

Figure 11 Basic configuration of the webGIS service DARES MAPPER, showing InSAR results and time-series over a mining area

Figure 12 shows a configuration of DARES MAPPER providing the accumulated vertical deformation from 19 August 2018 to 15 March 2019 in an area corresponding to a tailings dam, and the time-series evolution of the measurement point selected in the platform (highlighted with white circle). As illustrated, the cumulated deformation is represented jointly with the piezometric information for the same temporal span. The simultaneous analysis of the temporal evolution of the piezometric level and the deformation (two closest InSAR time-series) reveals a clear correlation pattern with a correlation coefficient of 0.92 . Despite the lack of continuous piezometric measurements (light blue line), it is clearly visible that both deformation trends (orange and dark blue lines) behave in a similar way with a detectable delay. 


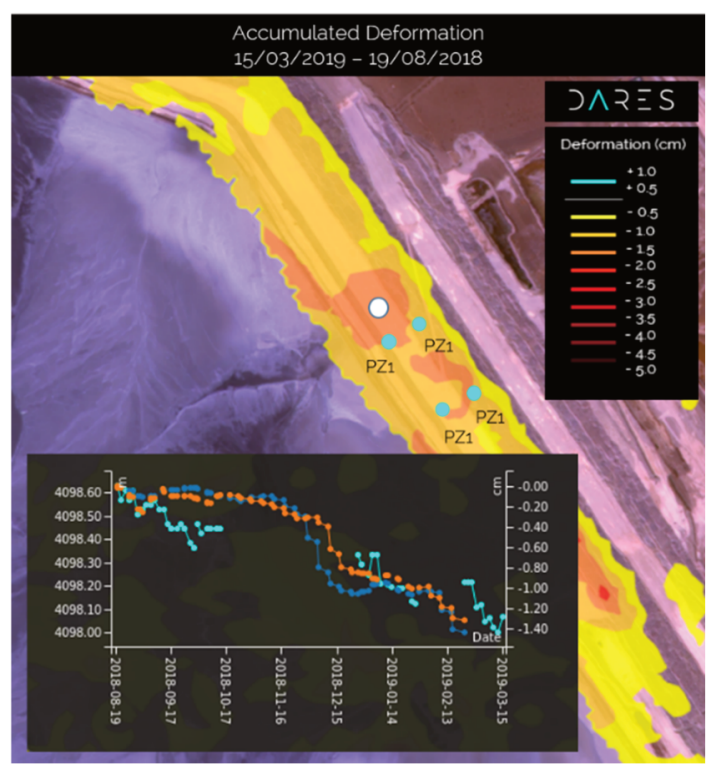

Figure 12 DARES MAPPER configuration showing the correlation between two closest InSAR time-series and piezometers, with a correlation index above 0.9

Finally, Figure 13 shows InSAR results compared to rainfall data giving by an in situ meteoritical station. The webGIS allows automatic integration (trough wifi data-loggers) and shows rainfall data superimposed to ground deformation time-series. As explained in the previous subsection, the automatic detection of acceleration periods and the correlation with in situ data (rainfall data in this case) allows possible interpretation and better understanding (or triggering) of slope instabilities.

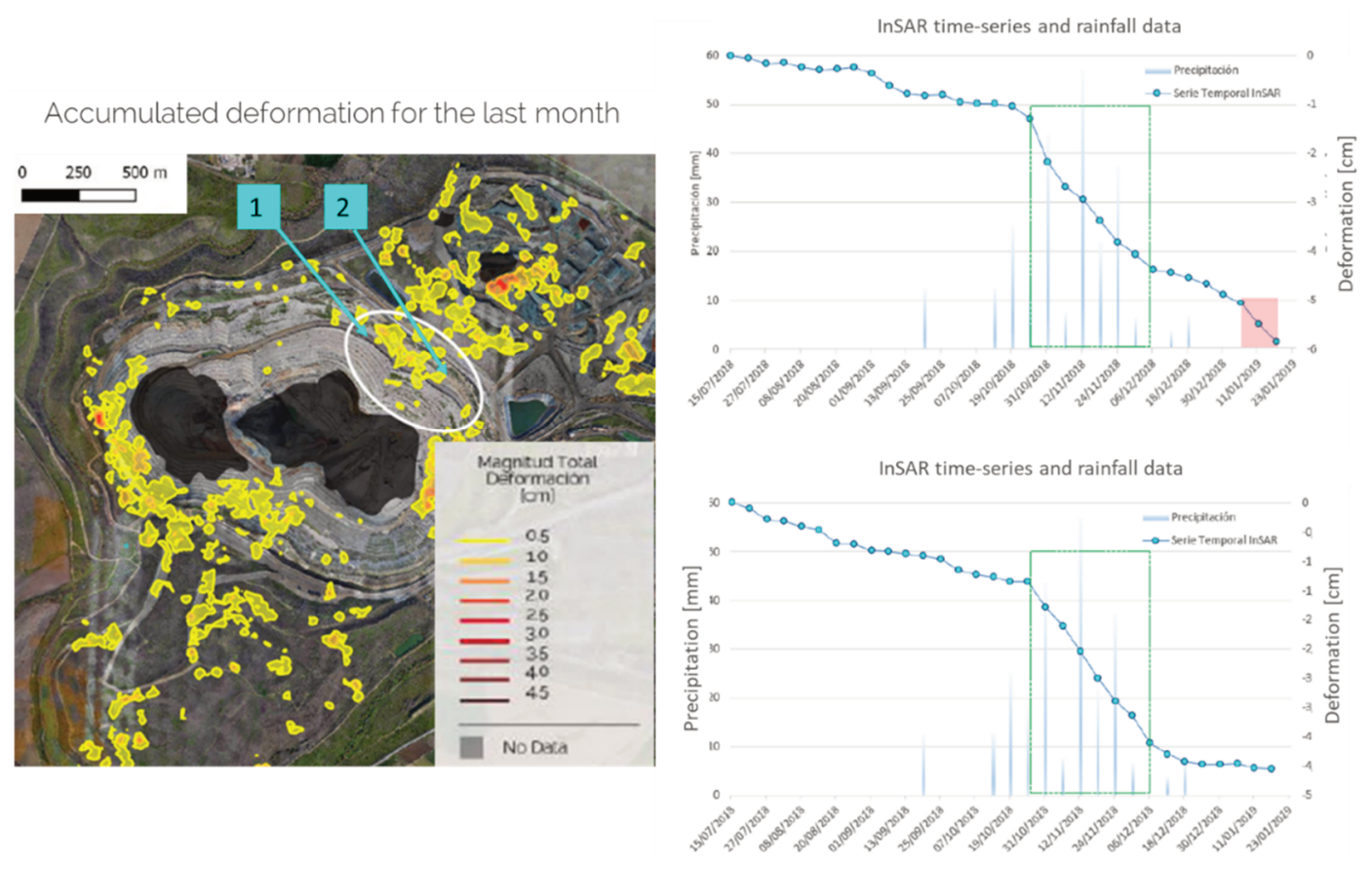

Figure 13 DARES MAPPER configuration showing the correlation between InSAR time-series and rainfall data

\section{Conclusion}

The increasing focus on mining safety brings with it an increasing awareness on the importance of reliable monitoring programs which include different surveying technologies. InSAR technology based on satellite radar data allows monitoring of ground displacements in mine areas with high precision and efficiency 
(remotely and for the whole area). InSAR can provide continuous monitoring of ground instabilities by providing information about accelerations on a weekly to monthly basis.

Loading the InSAR data in online webGIS servers, together with other geotechnical survey data gathered from in situ sensors, allows a better understanding of ground instabilities. Interactive analysis through cross-sections allows spatio-temporal analysis of ground deformation, along with specific transects that ease the understanding of the displacement dynamics. Additionally, automatic detection of ground displacement accelerations allows the systematic application of the inverse velocity technique, which in turn, provides a prediction of the date of a potential failure, based purely on a kinetic approach.

Several examples shown in this paper demonstrate the potential of InSAR data to detect geotechnical failures. The back-analysis over three events shows accelerations in ground deformations some weeks prior to the failure. The application of the inverse velocity technique provides an estimated time for the potential failure with errors of 3-5 days.

This paper also discusses that all these analyses and information can be obtained interactively by using online webGIS services, improving the user experience and allowing the comparison of different InSAR-derived products with in situ information. This paper shows examples of how data interpretation and trigger information of slope instabilities can be derived by combining InSAR data with ground piezometry and rainfall information. This type of combined analysis allows a better understanding of the phenomena and provides more complete information about the precursors of geotechnical instabilities, contributing to the decision-making process in the daily risk evaluation. The complete integration and automatic update of in situ instrumentation data in webGIS platforms, combined with InSAR information, is a complex task. Nevertheless, unification and centralisation of geotechnical databases, including InSAR, is one of the main future trends in geotechnical monitoring for mine operations.

\section{Acknowledgement}

We would like to acknowledge the European Space Agency and the Copernicus Satellite program for the availability of Sentinel-1 satellite data.

\section{References}

Arnaud, A, Adam, N, Hanssen, R, Inglada, J, Duro, J, Closa, J \& Eineder, M 2003, 'ASAR ERS interferometric phase continuity', Proceedings of 2003 IEEE International Geoscience and Remote Sensing Symposium, Institute of Electrical and Electronics Engineers, Piscataway, https://doi.org/10.1109/IGARSS.2003.1294035

Bernardino, P, Fornaro, G, Lanari, R \& Sansosti, E 2002, 'A new algorithm for surface deformation monitoring based on small baseline differential SAR interferograms', IEEE Transactions on Geoscience and Remote Sensing, vol. 40, issue 11, pp. 2375-2383.

Bürgmann, R, Rosen, PA \& Fielding, EJ 2000, 'Synthetic Aperture Radar interferometry to measure Earth's surface topography and its deformation', Annual Review of Earth and Planetary Sciences, vol. 28, pp. 169-209.

Carlà, T, Farina, P, Intrieri, E, Ketizmen, H \& Casagli, N 2018, 'Integration of ground based radar and satellite InSAR data for the analysis of an unexpected slope failure in an open-pit mine', Engineering Geology, vol. 235, pp. 39-52.

Ferretti, A, Prati, C \& Rocca, F 2001, 'Permanent Scatterers in SAR interferometry', IEEE Transactions on Geoscience and Remote Sensing, vol. 39, pp. 8-20.

Gabriel, AK, Goldstein, R.M \& Zebker, HA 1989, 'Mapping small elevation changes over large areas: Differential radar interferometry', Journal of Geophysical Research, vol. 94, pp. 9183-9191.

Hanssen, RF 2001, Radar Interferometry: Data Interpretation and Error Analysis; Kluwer Academic Publishers, Dordrecht.

Hooper, A, Zebker, H, Segall, P \& Kampes, B 2004, 'A new method for measuring deformation on volcanoes and other natural terrains using InSAR persistent scatterers', Geophysical Research Letters, vol. 31, https://doi.org/10.1029/2004GL021737

Lanari, R, Mora, O, Manunta, M, Mallorqui, JJ, Berardino, P \& Sansosti, E 2004, 'A small-baseline approach for investigating deformations on full-resolution differential SAR interferograms', EEE Transactions on Geoscience and Remote Sensing, vol. 42, pp. 1377-1386.

Massonnet, D \& Feigl, KL 1998, 'Radar interferometry and its application to changes in the Earth's surface', Reviews of Geophysics, vol. 36, issue 4, pp. 441-500.

Mora, O, Mallorqui, JJ \& Duro, J 2002, 'Generation of deformation maps at low resolution using differential interferometric SAR data.' Proceedings of 2002 IEEE International Geoscience and Remote Sensing Symposium, Institute of Electrical and Electronics Engineers, Piscataway, https://doi.org/10.1109/IGARSS.2002.1026745 
Exploitation of InSAR techniques as a support of in situ sensors

J Duro et al.

to improve safety and productivity in mining operations 